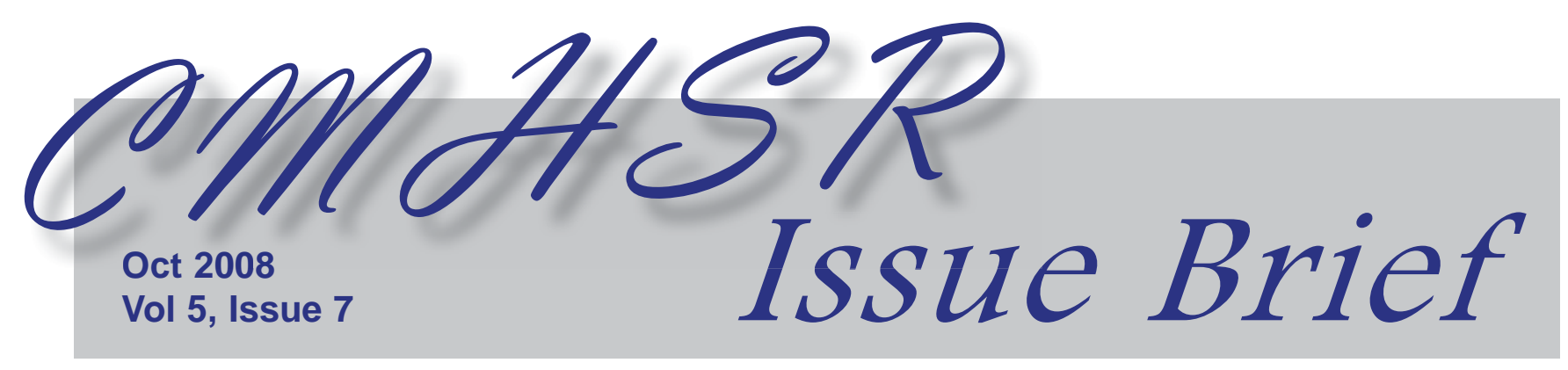

\title{
Family Networks Implementation Study: Integrating Rigor and Relevance in Effective Research Partnerships
}

Jodi Adams, MA, Joanne Nicholson, PhD, Susan Maciolek, MPP, Kate Biebel, PhD

$\mathrm{P}$ artnerships between academic researchers and public agencies are a common approach to the piloting, implementing, and testing of policy and practice initiatives. 1 These partnerships may benefit both parties by mustering support for rigorous research methods and increasing the relevance of research findings to the field.2,3 Although researchers have proposed collaborative partnership models and described vital characteristics of successful research partnerships, few have studied the conditions that effectively facilitate and sustain productive partnerships. ${ }^{1,4}$

Developing and sustaining productive relationships with public agency partners poses challenges to researchers. Organizational structure, culture, politics, and reward systems shape the motivations and priorities of both agency partners and academic researchers. ${ }^{5}$ In fact, these motivations and priorities may be at odds.3, 6 For example, fast-paced, crisis orientated public agencies may require rapid evaluations with findings most usefully described in one page of bulleted items or presented to senior staff. These work products may be unsuitable for professional publications or scientific presentations which are typically required of academic researchers. While the academic researcher's priority might be to make a scientific contribution, the agency's research priority may be driven by an immediate policy or practice need.

It is our contention that successful research partnerships with public agencies are based on a shared understanding and joint appreciation of the contingencies operating in each partner's domain. This appreciation may develop best in conditions of trust and respect, which contribute to good relationships and a growing portfolio of positive experiences. The outcomes of the most successful partnerships, generated in the context of a true learning community, are rewarding to both public agency representatives and researchers. Their shared priority and their commitment must be to integrate scientific rigor with real-world relevance. It is our experience in the Family Networks Implementation Study that pursuing one enhances, rather than precludes, the achievement of the other.

\section{The Family Networks Implementation Study (FNIS)}

Family Networks is a Massachusetts Department of Children and Families (MA/DCF) initiative to transform traditional purchased services, e.g., inhome services, specialized foster care and congregate care (group home settings), into an integrated child

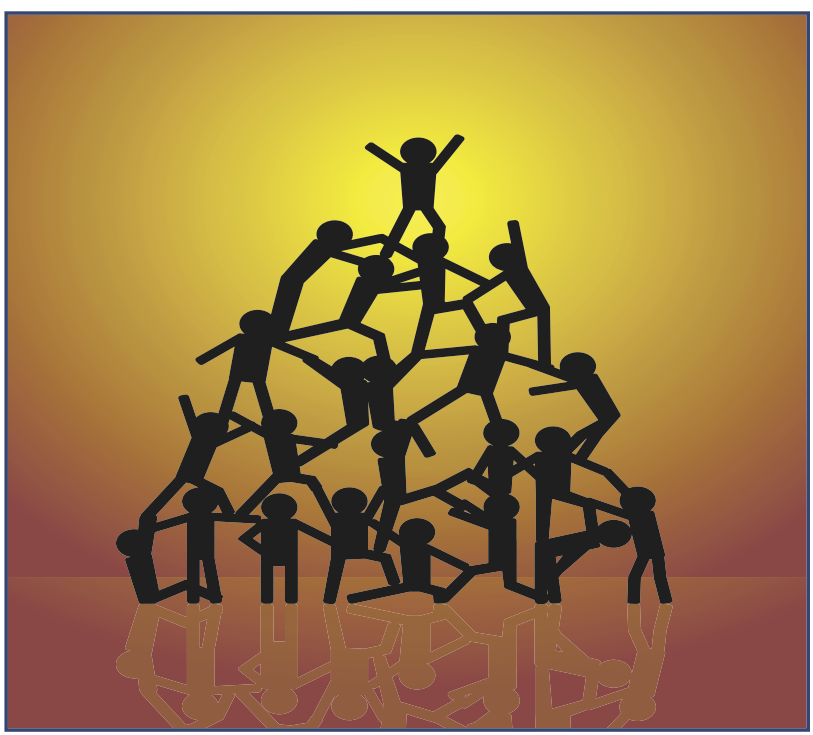


welfare service system for children, youth, and families. The FNIS is a mixed methods longitudinal study of the structures, processes and conditions that influence the implementation of Family Networks, being conducted by partners from CMHSR and the MA/DCF. CMHSR researchers have a long-standing commitment to research on questions generated in real-world contexts, with implications for policy and practice. MA/DCF represents a new research relationship for CMHSR, requiring the development of strategies and mechanisms for building and sustaining the partnership.

\section{Shared Power Leads to Trust}

Shared project management and the active involvement of stakeholders have contributed to trust and enhanced commitment to the FNIS. The study is co-directed by CMHSR and MA/DCF project leaders who share power in all aspects of the study. Two functional project teams include representatives from MA/DCF and CMHSR. The Project Management Team (PMT) informs the study design, recommends research methods and measures, specifies the data collection schedule, and deals with study logistics. The Study Advisory Team (SAT) includes an array of Family Networks stakeholders (e.g., provider agency and MA/DCF regional and area office representatives) who assist in refining research questions, advise regarding sampling and data collection strategies, interpret findings, review deliverables and recommend feedback.

\section{Shared Knowledge \& Expertise Lead to Respect}

Mutual respect develops when partners share knowledge and expertise to refine study procedures, reduce study burden, and promote the likelihood of study success. For example, the PMT created a library of handouts about research design and methods to inform SAT members unfamiliar with research strategies. MA/DCF partners and stakeholders share information about their experiences in the field, and in previous research and evaluation efforts that have been more or less successful. They provide access to key informants, agency managers, and community networks of providers who inform and participate in the study.

\section{Communication Leads to Shared Understanding}

Open communication facilitates the development of mutual mentoring relationships, which allow study partners and stakeholders to learn about and adapt to the time frames, priorities, contingencies, and values of each other's dynamic organizations. FNIS partners and stakeholders spend a great deal of time discussing their respective organization's roles, responsibilities, languages, norms, and physical environments. Rather than compromising research rigor, this shared understanding allows for a transparent alignment of ideas and values between partners. For example, a MA/DCF manager may be reassured that research sampling strategies are consistent with strategies they might use to insure equal representation.

\section{Shared Experiences Lead to a Learning Community}

The FNIS provides MA/DCF partners with the opportunity to reflect upon agency activities and study findings, while providing CMHSR partners with the opportunity to contextualize research results. The FNIS design is a collaborative, iterative process in which findings from each phase of research are used to develop subsequent phases. During this process, FNIS partners engage in conversations about factors related to rigor, and address questions about relevance in a dynamic, changing public agency.

\section{Integrating Rigor and Relevance}

The partnership of public agency partners with academic researchers can lead to clearer strategies and more relevant findings for maintaining rigor in community research. A shared understanding of the contingencies operating in each domain can contribute to the building of productive relationships and a learning context in which rigorous research can be conducted and have real-world impact.

\section{References}

1. Suarez-Balcazar, Y., Harper, G. W., \& Lewis, R. (2005). An interactive and contextual model of community-university collaborations for research and action. Health Education छ' Behavior, 32(1), 84-101.

2. Lindamer, L. A., Lebowitz, B. D., Hough, R. L., Garcia, P., Aquirre, A., et al. (2008). Improving care for older persons with schizophrenia through an academiccommunity partnership. Psychiatric Services, 59(3), 236-239.

3. Kaufman, J. S., Crusto, C. A., Quan, M., Ross, E., Friedman, S. R., et al. (2006) Utilizing program evaluation as a strategy to promote community change: evaluation of a comprehensive, community-based, family violence initiative. American Journal of Community Psychology, 38, 191-200.

4. Tseng, V. (2007). Studying the use of research evidence in policy and practice. William T. Grant Foundation 2007 Annual Report, 12 -19.

5. Harper, G. W., Bangi, A. K., Contreras, R., Pedraza, A., Tolliver, M., \& Vess, L. (2004). Diverse phases of collaboration: Working together to improve communitybased HIV interventions for adolescents. American Journal of Community Psychology, 33(3-4), 193-204.

6. Jones, L., \& Wells, K. (2007). Strategies for academic and clinician engagement in community-participatory partnered research. JAMA, 297(4), 407-410.

Visit us on-line at www.umassmed.edu/cmhsr Send Comments to CMHSR@umassmed.edu 